Document downloaded from:

http://hdl.handle.net/10251/178924

This paper must be cited as:

Bouslama, R.; Jordán, J.; Heras, S.; Amor, NB. (2020). Strategies in Case-Based Argumentation-Based Negotiation: An Application for the Tourism Domain. Springer. 205217. https://doi.org/10.1007/978-3-030-51999-5_17

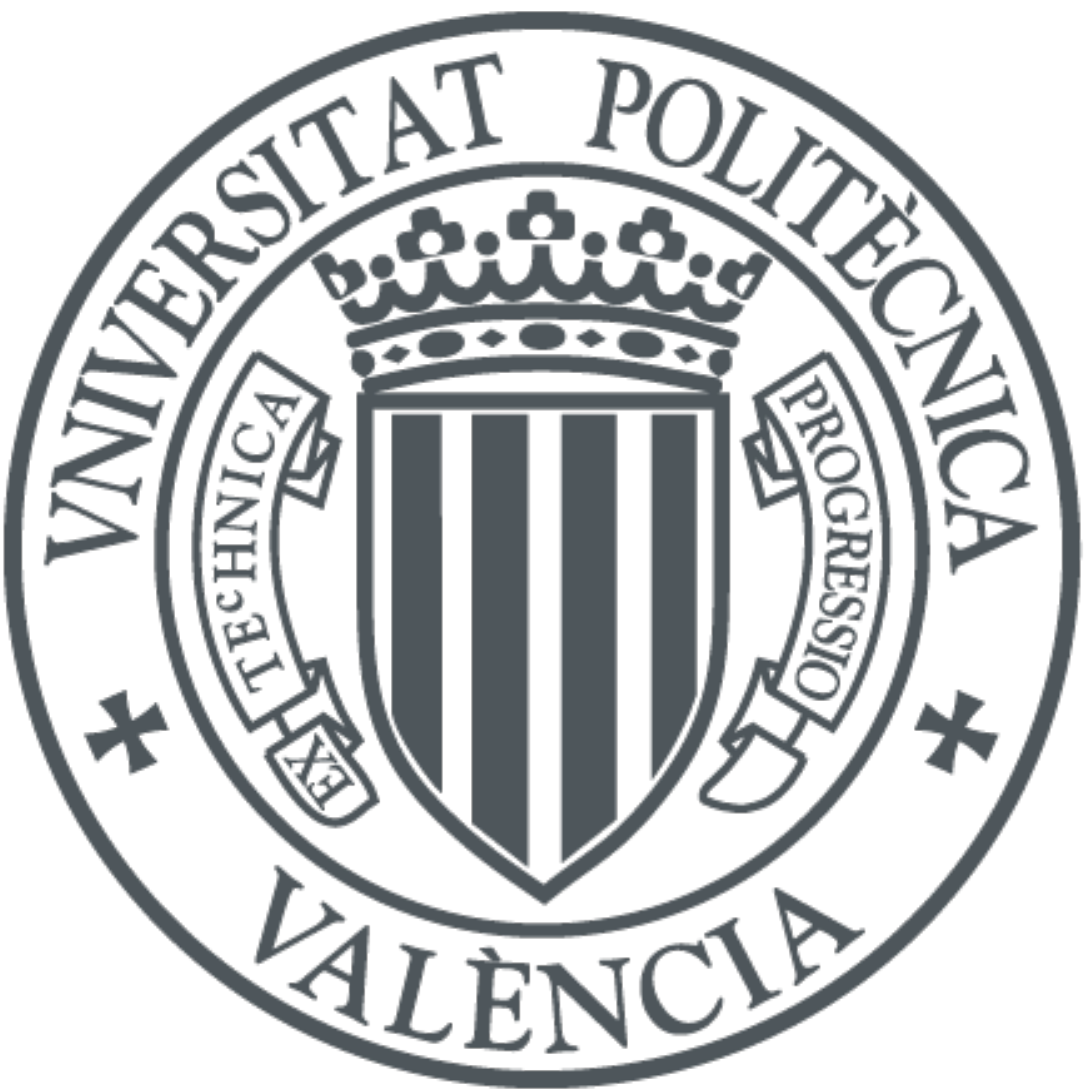

The final publication is available at

https://doi.org/10.1007/978-3-030-51999-5_17

Copyright Springer

Additional Information 


\title{
Strategies in Case-Based Argumentation-Based Negotiation: An application for the tourism domain
}

\author{
Rihab Bouslama ${ }^{1}$, Jaume Jordán ${ }^{2[0000-0003-0400-9136], ~ S t e l l a ~}$ \\ Heras $^{2[0000-0001-6212-9377]}$, and Nahla Ben Amor ${ }^{1}$ \\ 1 LARODEC, ISG, Université de Tunis, Tunis, Tunisia \\ rihabbouslama@yahoo.fr, nahla.benamor@gmx.fr \\ 2 Valencian Research Institute for Artificial Intelligence (VRAIN), \\ Universitat Politècnica de València, Camino de Vera s/n, 46022 Valencia, Spain. \\ \{jjordan, sheras\}@dsic.upv.es http://vrain.upv.es/
}

\begin{abstract}
Negotiation is a key solution to find an agreement between conflicting parties especially during the purchase journey. This paper treats the negotiations between a travel agency and its customers in the domain of tourism. Both automated negotiation and argumentation are gathered to create a framework for automated agents, presenting a travel agency and its customers, to negotiate a trip and exchange arguments. Agents take advantage of their past experiences and use Case-Based Reasoning to select the best strategy to follow. We represent agents using two types of profiles, Argumentative profile that represents agents' ways of reasoning and Preference profile that embodies customers' preferences in the domain of tourism.
\end{abstract}

Keywords: Automated Negotiation · Argumentation · Case-Based Reasoning $\cdot$ Strategy $\cdot$ Tourism

\section{Introduction}

The e-tourism is growing over the last decade [13], implying an increase of the percentage of customers that book a trip online. Hence, it is necessary to have an efficient booking system that takes into account the whole process of booking, including the negotiation between customers and travel agencies. A trip may be negotiated based on different criteria (e.g., destination), where customers try to minimize their costs and get the best offer that matches their preferences, and the travel agency tries to maximize its profits and satisfy its customers.

Automated negotiation is an important sub-field of the Artificial Intelligence (AI) domain. Proposals mainly concern negotiation protocols, negotiation frameworks, and reasoning mechanisms in the context of Multi-Agent Systems (MAS) [12]. Other research works went beyond exchanging offers between agents and proposed the exchange of arguments as additional information to enhance the negotiation process and include persuasion $[7,16]$. Argumentation is widely studied in MAS as a mean to solve conflicts between agents with positions [14]. 
Another interesting research area of AI is Case-based Reasoning (CBR) [1], which has been studied in both argumentation and negotiation. Few attempts included CBR in Argumentation-Based Negotiation (ABN) frameworks such as the preliminary work of Sycara [20], where she proposed the PERSUADER system that plays the role of a mediator between a company and its trade union in the domain of labour management. CBR was also used in the domain of resources allocation to select a negotiation strategy from the set of past negotiations of the agents [19]. A generic framework for ABN using CBR was proposed for bilateral settings [5]. Then, an improved version for mediated multilateral negotiations was presented in [6]. Both of these works were based on the work proposed in [10], where authors presented a case-based argumentation approach for MAS.

An important aspect of automated negotiation is agents' strategies. They are influenced by many factors such as the negotiation domain, goals, and agents' profiles. [14] discusses different strategies that are originally studied in the social science in the domain of energy market. In particular, it focuses on two groups of strategies: concession making and problem solving where agents start from an opening position (extreme, reasonable, or modest) and make a sequence of concessions (large, moderate, or small). In an ABN context, [8] defines a strategy as a set of tactics that are short time moves to pursue a high-level goal. They propose three tactics: Boulware, Conceder, and LastMinuteTactic. One way to select the best strategy is to use decision trees as proposed in [9]. Another approach uses CBR [11], where strategies are based on agents' profiles that define their attitude towards the generated and received arguments.

Most of the frameworks that include computational argumentation ensure reasoning by applying a set of inference rules, which requires an explicit model of the domain. In fact, in the domain of tourism it is not possible to define the rules in advance. Thus, a case-base representation of agents' reasoning makes the framework more dynamic, and agents' knowledge easier to maintain.

In this work, we propose an ABN system in the domain of tourism. The system is dedicated for travel agencies to help them in the negotiation process with their customers, represented as automated agents. We define preference profiles that explain the customers' desires and argumentative profiles that illustrate the travel agency's and its customers' ways of arguing. Considering the benefits of using a CBR, both negotiation parties will follow a case-based strategy generation. CBR helps agents to select the argument to put forward at each step of the negotiation and thus, devise the dialogue strategies based on their experiences.

The paper is organized as follows. Section 2 presents the architecture of the proposed framework and its main components. Section 3 discusses the negotiation protocol and the strategical reasoning followed by agents. Finally, Section 4 summarises the paper and proposes future work.

\section{Case-Based Argumentation-Based Negotiation}

This work aims to propose an ABN framework in the domain of tourism to support trip negotiations of a travel agency and its customers where the exchange 
of arguments between agents is devised by the CBR. Formally, the proposed ABN framework is defined as a tuple $A B N=\left\langle A_{0}, A, O, \operatorname{Arg}\right\rangle$ where $A_{0}$ is the travel agency agent, $A$ : the set of $n$ customer agents such that $A=\left\{A_{1}, \ldots, A_{n}\right\}, O=$ $\left\{O_{1}, \ldots, O_{n}\right\}$ is the set of all possible offers for $n$ customer agents. Concretely, for an agent $i$ with $m$ offers, $O_{i}=\left\{o_{i 1}, \ldots, o_{i m}\right\}$ and $\operatorname{Arg}=\left\{\operatorname{Arg}_{1}, \ldots, \operatorname{Arg}_{n}\right\}$ is the set of all arguments exchanged between the $A_{0}$ and its $n$ customer agents. For an agent $i, \operatorname{Arg}_{i}=\left\{\arg _{i 1}, \ldots, \arg _{i l}\right\}$, where $l$ is the number of arguments.

The global architecture is depicted in Figure 1 and its main components (i.e., agents, knowledge resources, arguments) are detailed below.

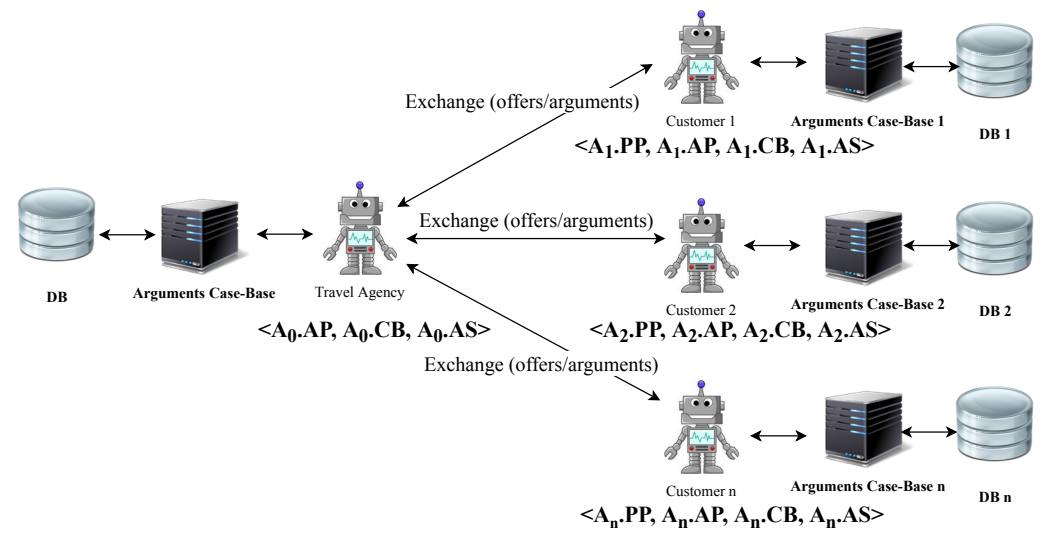

Fig. 1: The global architecture of the proposed framework

\section{$2.1 \quad$ Agents}

The framework is conceived for a MAS that supports agents with different behaviours. Agents are defined based on their profiles that rule their behaviour and importantly, their reasons to accept or reject an offer or an argument. Given a travel agency $\left(A_{0}\right)$ and a customer $\left(A_{i}\right)$ we define an argumentative profile $A_{0} . A P$ (resp. $A_{i} \cdot A P$ ) for both agents, that characterizes the way they negotiate and argue. For the customers, we also define a preference profile $\left(A_{i} . P P\right)$, which embodies their preferences regarding a trip.

Argumentative Profile The argumentative profile of an agent defines the way it persuades, accepts or rejects offers and arguments. To define this profile, we adapt the profiles proposed in $[3,11]$ to an $\mathrm{ABN}$ setting as follows:

- Agreeable: the easiest agent to convince in the negotiation. Prefers short time negotiations. Accepts whenever possible. Agrees on an argument if she does not have an attack argument. When she loses a round (we have at least one round), she accepts the position of the counter party only if it is in her list of potential offers. This type of agent makes big concessions. 
- Open minded: an average negotiator agent. Not influenced by time. Agrees on an argument if she does not have an attack argument. If she loses a round, she will not accept an offer if it not her current preferred one. She will make small concessions by proposing the next most preferred offer.

- Argumentative: the strictest negotiator agent. Prefers long negotiations with a maximum of utility. Agrees on an argument if she does not have an attack argument. If she loses a round and she still have arguments in her casebase, she will insist on her previous offer. Otherwise, she will make small concessions (next most preferred offer). She will not accept an offer if it is not her current preferred one even if it is in her list of preferred offers.

Preference Profile The preference profile is domain dependent and defines customers' preferences regarding a trip. In fact, a trip has a set of features that may be classified to fundamental and optional. Besides the price and the destination, features as season, weather, country's safety rank, health and hygiene rank, and country's global rank represent fundamental features that interest all type of agents with different degrees. Optional features as the existence or not of shopping areas and monuments, depend on agent's preference profile. Inspired from the work presented in [15], we propose the following profiles of customers (using examples of optional features) that embody the travel personae:

1. Photographer (agent looking for nice places): she may be influenced by features as buildings for sight seeing, natural parks, monuments, and mountains.

2. Popular cities seeker (agent looking for typical holiday destinations): she may be influenced by features as means of transportation, monuments, museums, and local markets.

3. Adventure seeker (agent looking for adventure holidays): she is interested by adventure activities and active tourism possibilities in these places (e.g., mountains, jungle, desert).

4. Parties seeker (agent looking for parties and fun activities): she may be influenced by features as concerts, restaurants, and coffee shops.

5. Fashion seeker (agent looking for shopping areas): she may be influenced by features as shopping area and local markets.

6. Relaxation seeker (agent looking for relaxation): she may be influenced by features as hotel's accommodation and hotel's number of stars.

7. Culture seeker (agent looking for cultural attractions): she may be influenced by features as mosques, cathedrals, synagogues, monuments, and museums.

8. Food seeker (agent looking to try different food): she may be influenced by features as local markets, restaurants, coffee shops, and hotel accommodation.

\subsection{Knowledge Resources}

In our framework we make use of argument case-bases and argumentation schemes as knowledge resources to generate and manage arguments, following the approach proposed in $[5,10]$. Case-Based Reasoning (CBR) is a methodology based on the idea that similar cases have similar solutions [1]. This reasoning is so 
similar to human behaviour during a negotiation where they use their past experiences to justify their positions.

From this perspective, we propose an Arguments Case-Base (ACB) to be the main knowledge resource used by agents to make decisions (e.g., generating and selecting a specific argument). The travel agency has its own ACB denoted by $A_{0} . C B$. Similarly, a customer $i$ has her own ACB denoted by $A_{i} . C B$. Then, with this resource we can follow a CBR approach and make possible for the agents to learn about different profiles, the type of arguments that they usually accept (resp. reject), and at which time of the dialogue a specific argument was accepted. The ACB is composed of several cases that embody an agent's argumentation experience (store previous arguments in the form of cases). Therefore, each exchanged argument in the dialogue is retained in the case-base. The components of one argument case are:

- Profile: both preference profile as well as argumentative profile of the opponent are stored. This information helps agents to learn about their opponents and to know which kind of arguments they accept (resp. reject).

- Argument Type: may be any type of arguments that will be further discussed (e.g., explanation, reward). This information helps the agent to learn the type of arguments that is usually accepted by each profile.

- Argument Conclusion: the conclusion of the argument (commonly, the outcome of the offer that it supports or attacks).

- Argument Acceptance: the final decision (i.e., acceptance or rejection) on each argument is saved to help argument selection.

- Acceptance time: the time of acceptance (e.g., at the beginning, at the end) of the argument is also important. It helps agents to select the appropriate argument at each period of the dialogue, since it can denote patterns, such as that a specific type of argument often results in shorter dialogue.

- Success Rate: indicates how many times a given argument was accepted in a given time by a given agent. Indeed, arguments with the highest Success Rate are the arguments that have more chance to be accepted.

By storing these information, agents are able to select the best strategy to follow in each situation. Table 1 shows an example of the information stored in an ACB. By the argument case AC1 we can infer that popular cities seekers with an argumentative profile would quickly accept arguments to support offers for Paris at a price of $100 €$ if the agency includes breakfast for free, even when they commonly engage in large negotiations. However, they would reject the same argument, and hence the underlying offer, if the price increases to $200 €$.

The second knowledge resource is Argumentation Schemes (AS). They represent patterns of common human reasoning. The work of Walton [21] presented 29 different AS taking the form of a set of premises, a conclusion and a set of critical questions (possible ways of attacking the underlying argument). From this set of schemes, we selected a subset which especially captures the usual mode of reasoning in the tourism domain:

- Argument from Popular Opinion (APO): captures the fact that humans tend to believe a thing true if the majority hold this opinion. In our travel domain, 
this stands for the fact that if most customers accept a given offer in a given circumstances then, it is a good reason for other customers to accept it.

- Argument from Popular Practice (APP): is a variant of the above, representing the fact that humans are willing to do what most people do. In the travel domain, the travel agency can convince its customers by referring to popular practices. Indeed, similar travellers tend to do similar activities.

- Argument from Expert Opinion (AEO): captures the reasoning by which humans accept as true the opinions of an expert. Travel agencies work with experts from different fields. Thus, appealing to their knowledge is a way to convince customers. For example, if the travel agency appeals to information coming from the world economic forum tourism report, customers may trust accepting the offer.

- Argument from Waste (AW): captures the behaviour by which humans try not to waste the work done. Spending a long time for the negotiation may be expensive for the travel agency and for the customers. In such cases, accepting a given offer may prevent the negotiation parties from losing money.

Therefore, AS can be combined with the information stored in the ACB to generate arguments that represent the underlying line of reasoning that each scheme captures. For instance, in a negotiation with a popular cities seeker with an argumentative profile, and in view of the $\mathrm{AC} 1$ represented in Table 1, by the APP AS we can generate an argument to support an offer (Paris at $100 €$ per night), since it is common for this type of agents ( $80 \%$ success rate) to quickly accept this type of offer, so the travel agency could speed up the negotiation.

\begin{tabular}{l|c|c|c|c|c|c}
\hline AC ID & Profile & Arg. Type & $\begin{array}{c}\text { Conclu- } \\
\text { sion }\end{array}$ & $\begin{array}{c}\text { Arg. } \\
\text { Acceptance }\end{array}$ & $\begin{array}{c}\text { Acceptance } \\
\text { Time }\end{array}$ & $\begin{array}{c}\text { Success } \\
\text { Rate }\end{array}$ \\
\hline AC1 & $\begin{array}{c}\text { Argumentative+Popular } \\
\text { Cities Seeker }\end{array}$ & $\begin{array}{c}\text { Reward (Ac=Breakfast } \\
\text { included for free) }\end{array}$ & $\begin{array}{c}(\text { Paris, } \\
100 €)\end{array}$ & Accept & Beginning & $80 \%$ \\
\hline AC2 & $\begin{array}{c}\text { Argumentative+Popular } \\
\text { Cities Seeker }\end{array}$ & $\begin{array}{c}\text { Reward (Ac=Breakfast } \\
\text { included for free) }\end{array}$ & $\begin{array}{c}(\text { Paris, } \\
200 €)\end{array}$ & Reject & Beginning & $90 \%$ \\
\hline \multicolumn{7}{r|}{ Table 1: Example of an Argument Case-Base } \\
\hline
\end{tabular}

\subsection{Arguments}

Arguments represent the persuasion part of the negotiation which influences agents' initial spaces of acceptance. Several classifications of arguments are evoked in the literature. Arguments may be classified as practical arguments expressing facts used to support an offer or epistemic arguments presenting agents believes. Overall, arguments may entail different purposes [10,11]:

- Support: arguments that support an offer proposed by an agent.

- Attack: rebut and undercut arguments where the former attacks the counter party's offer (i.e., the conclusion of an argument) and the latter attacks the premises of the counter parties' arguments.

Another threefold classification highly used in the argumentation community was proposed in [18] where we distinguish threats, rewards and appeals. Amgoud 
and Prade [4] propose a close variant of this classification by saving threats and rewards and defining classical ABN arguments, the so-called explanations, arguing that the different forms of appeals can be modeled in this class. Following their taxonomy, our framework includes explanations, threats and rewards.

Besides the fact that explanations, threats and rewards are very common in the argumentation field, they illustrate the type of arguments usually exchanged between sellers and buyers. Following the definition of an argument proposed in [11] we define an explanation argument as:

Definition 1 (Explanation argument). $\operatorname{Arg}=\langle\phi, S\rangle$, where $\phi$ is the conclusion of the argument, and $S$ is the support set of an argument.

We define threat and reward arguments as follow:

Definition 2 (Threat argument). Arg $=\langle\phi, S, A c\rangle$, where $\phi$ is the conclusion of the argument, $S$ is the support set of an argument, and Ac is an action that threatens the counter party and violates her goals.

Definition 3 (Reward argument). Arg $=\langle\phi, S, A c\rangle$, where $\phi$ is the conclusion of the argument, $S$ is the support set of an argument, and $A c$ is an action that promotes a reward for the counter party.

The support set can consist of different elements, depending on the argument purpose. On the one hand, if the argument entails a support, the support set is the set of features (premises) that represent the context of the domain where the argument has been proposed (e.g. features of the offer) and optionally, any knowledge resource used by the proponent to generate the argument (argumentation schemes and argument-cases). On the other hand, if the argument is an attack argument, the support set can include any of the allowed attacks in our framework (distinguishing premises (DP) or counter-examples (CE)).

Thus, a DP is a premise that distinguishes two offers (represents a feature with a different value for these offers). A CE in our framework is an offer that includes the same features than another offer, but promote different outcomes.

\section{Argumentation-Based Negotiation protocol}

In this section, we present the $\mathrm{ABN}$ protocol that governs the negotiation between agents. Although we focus on a bilateral negotiation between the travel agency and each one of its customers, the same process may be executed simultaneously with $n$ customers (i.e., concurrent negotiation [2]). However, the most common operation of a travel agency is to engage in one to one negotiations (even when the booking is for a group, there is usually one representative of the group). The protocol includes two phases: (i) negotiation phase (i.e., exchange of offers) and (ii) argumentation phase (i.e., exchange of arguments).

Algorithm 1 describes an overview of the whole negotiation phase. First, the travel agency $\left(A_{0}\right)$ generates a set of possible offers based on the customer $\left(A_{i}\right)$ preferences (line 3 ). Then, $A_{i}$ challenges the offer that she prefers and waits for 


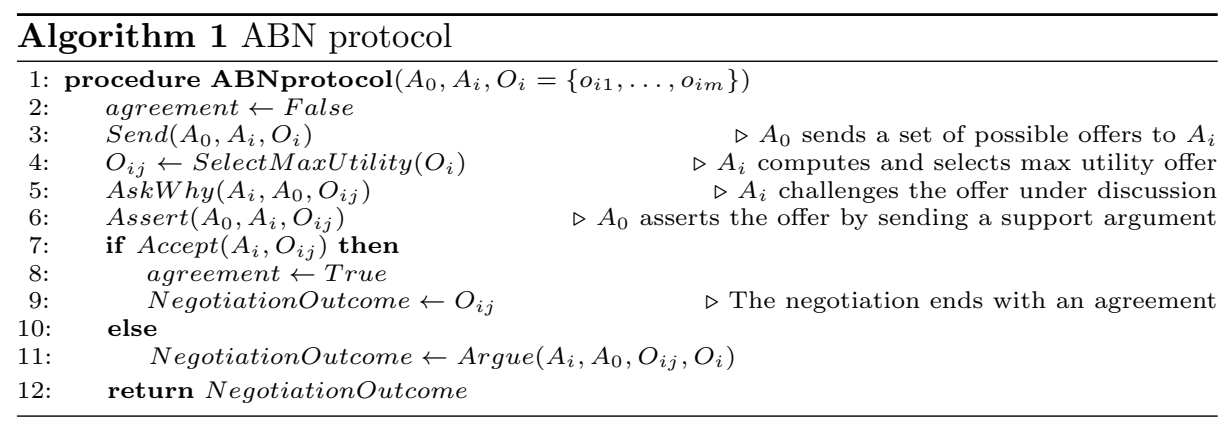

explanations from $A_{0}$ (lines 4-5). In her turn, $A_{0}$ sends arguments to support her offer (line 6). In this step, two cases are possible: $A_{i}$ accepts $A_{0}$ 's offer (lines 7-9), or $A_{i}$ attacks $A_{0}$ 's offer and enters in the argumentation process (line 11).

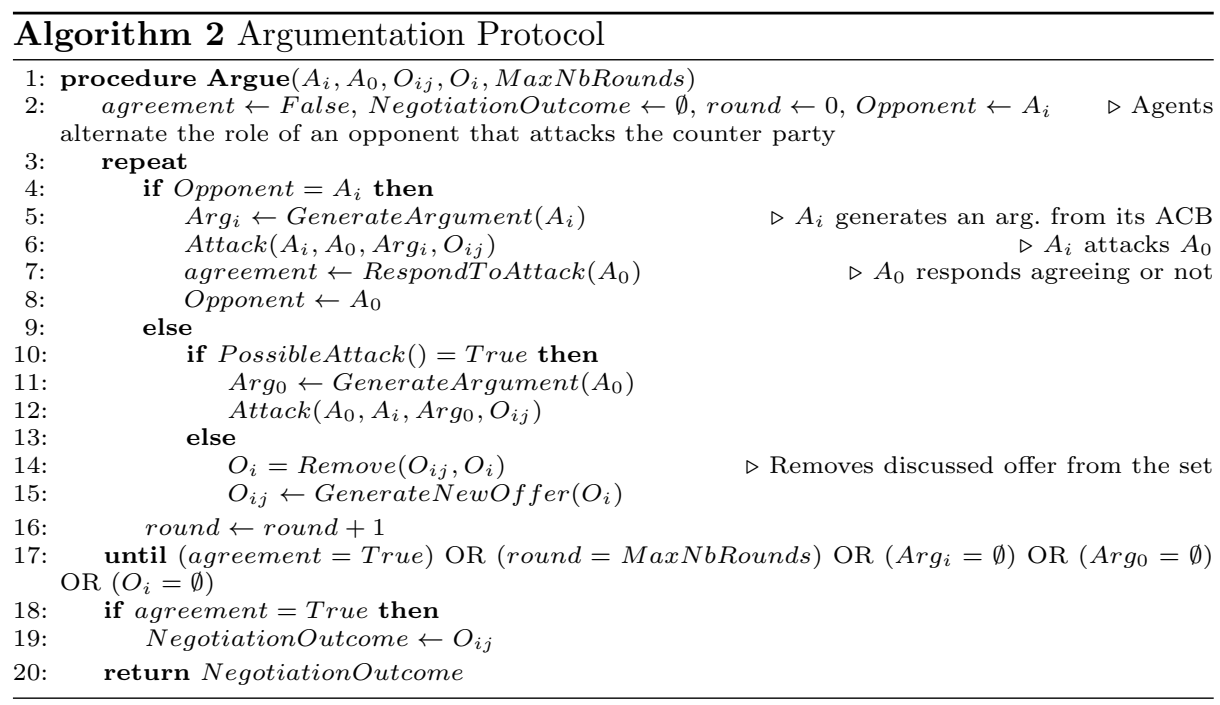

Algorithm 2 outlines the argumentation phase. Agents follow an alternating offers protocol similar to the one proposed in [5] in order to attack or support an argument (resp. an offer), or propose a new offer.

\subsection{Offers generation and evaluation}

As highlighted in Algorithm 1, $A_{0}$ is in charge of generating the set of possible offers $\left(O_{i}\right)$ based on $A_{i}$ 's preferences. To do so, she uses a data base that contains a set of features describing different trips.

Definition 4 (Offer). $o=\langle F, \Theta\rangle$, where $F=\left\{f_{1}, \ldots, f_{n}\right\}$ is the set of $n$ features characterizing an offer, and $\Theta$ is the outcome of the offer. 
In Definition 4, an offer is characterised by a set of features $F$. Each agent distributes a preference order over these features where $f_{1} \prec_{i} f_{2}$ indicates that the feature $f_{2}$ is more preferred than feature $f_{1}$ for agent $i$. For instance, hotel_pension $\prec_{A_{1}}$ safety means that for $A_{1}$ safety is more important than hotel_pension. $\Theta$ represents the features price and destination. For example, the first row of Table 2 is an offer where SafetyRank, Period and Weather are the set of features, and the outcome is Paris for $100 €$ per night.

\begin{tabular}{ccccc}
\hline Destination & Safety Rank & Period & Weather & Price per Day \\
\hline Paris, France & 5.7 & December Cold/Rainy & $100 €$ \\
Cancun, Mexico & 4.2 & July & Hot & $80 €$ \\
\hline
\end{tabular}

Table 2: Example of data

Agents evaluate offers using their utility functions that embody the preferencebased aspect of our framework. In fact, each agent has a different satisfaction value that she gets from each feature and thus from a given offer.

Definition 5 (Satisfaction of Offer). The satisfaction from a feature for an agent $A_{i}$ is defined using a satisfaction function: $A \times F \rightarrow \mathbb{R}^{+}$, where the satisfaction from the whole offer is: satisfaction $\left(A_{i}, o\right)=\sum_{k=1}^{n} \operatorname{satisfaction}\left(A_{i}, f_{k}\right)$.

The satisfaction from a feature represents its worth for an agent. For instance, for a customer $A_{1}$ the preference order over features is concerts $<_{A_{1}}$ accommodation. Since, one feature may have different values, then the agent gets different satisfaction such as satisfaction $\left(A_{1}\right.$, accommodation = allinclusive $)$ $=10.5$ while satisfaction $\left(A_{1}\right.$, accommodation $=$ breakfastandbed $)=6.1$. This means $A_{1}$ is more satisfied with an all inclusive accommodation. However, each offer has a cost for the agent (i.e., price):

Definition 6 (Cost of Offer). A cost of an offer o is defined using a cost function: $A \times o \rightarrow \mathbb{R}^{+}$, where the cost of the offer represents the price to pay for that offer: $\operatorname{cost}\left(A_{i}, o\right)=\operatorname{price}(o)$.

As in [17], we define the utility function in terms of satisfaction and cost:

Definition 7 (Utility of Offer). The utility of an offer o for an agent $A_{i}$ is: $u\left(A_{i}, o\right)=\left(\operatorname{satisfaction}\left(A_{i}, o\right)-\operatorname{cost}\left(A_{i}, o\right)\right) \times \delta_{A_{i}}$

In the above definition, $\delta$ is a discount factor that represents the influence of time on agents' utilities. The level of influence differs according to agents' profiles.

\subsection{Argument generation and evaluation}

Agents have a data base with information about several trip destinations. Table 2 depicts an example of these information that concern two destinations, their safety rank, the period of the trip, the weather and the price per day. 
At first, arguments are constructed using this knowledge base and by taking into consideration the context of the negotiation. For instance, if agents are negotiating Paris as a destination, an explanation argument as: $\langle$ (Paris, 100€), safety rank $=5.7\rangle$ may be generated. After generating the arguments based on the negotiation context, they are stored in case bases. Besides the argument itself, the information stored in the case bases (Section 2.2) helps agents to select the best argument to send in new negotiations. Indeed, the selection of a strategy is ensured following the CBR. Actually, $A_{0}$ and $A_{i}$ will challenge each other. More precisely, the persuasion phase is based on two parts:

Challenging the counter party's argument by sending:

-Rebut argument: may be sent to (1) criticize the offer promoted by the attacked argument by sending an explanation (e.g., CE), (2) send a reward or threat to the counter party to change her offer or to accept an offer or (3) send an AS.

-Undercut argument: may be sent to (1) criticize one or many features of the attack argument, (2) ask for more explanations on the offer promoted by the argument, (3) add additional information that were missed (DP). If agents need more explanation about the offer, they will ask for more information from the counter party (will be sent as additional features).

Responding to a challenge depends on the received argument:

-Response to a rebut: by (1) proposing another offer or (2) sending an AS.

-Response to an undercut: by (1) sending an explanation, (2) proposing another offer, (3) sending an AS or (4) sending Threat or Reward.

The choice of which argument to send from the above list represents the strategy that an agent follow which is based on her profile and her $A C B$.

\subsection{Illustrative Example}

Figure 2 depicts an ABN dialogue between the travel agency $A_{0}$ and a customer $A_{i}$. Agents' argumentative profiles are: $A_{0} \cdot A P=$ OpenMinded while $A_{i} \cdot A P=$ Argumentative. For the customer: $A_{i} \cdot P P=$ PopularCitiesSeeker. $A C B_{0}$ (resp. $A C B_{i}$ ) and $A S_{0}$ (resp. $A S_{i}$ ) are the travel agency (resp. customer) knowledge resources. Table 3 depicts $A_{0}$ and $A_{i}$ case bases where besides $A C 3$, the travel agency has in her CB the cases presented in Table 1. $A_{i}$ starts by requesting a trip proposition from $A_{0}$ indicating that she is looking for a safe country with a good hygiene rank where she can find museums and monuments. $A_{0}$ sends two possible offers (presented in Table 2).

\begin{tabular}{|c|c|c|c|c|c|c|c|}
\hline ACB ID & AC ID & Profile & Arg. Type & $\begin{array}{l}\text { Con- } \\
\text { clusion }\end{array}$ & $\begin{array}{l}\text { Arg. Ac- } \\
\text { ceptance }\end{array}$ & $\begin{array}{c}\text { Accep- } \\
\text { tance } \\
\text { Time }\end{array}$ & $\begin{array}{l}\text { Success } \\
\text { Rate }\end{array}$ \\
\hline$A C B_{0}$ & $\mathrm{AC} 3$ & $\begin{array}{c}\text { Argumentative+Popular } \\
\text { Cities Seeker }\end{array}$ & $\begin{array}{c}\text { Explanation }(\mathrm{AS}= \\
\mathrm{APO})\end{array}$ & $\begin{array}{c}\text { (Paris, } \\
100 €)\end{array}$ & Accept & Beginning & $80 \%$ \\
\hline$A C B_{i}$ & $\mathrm{AC} 1$ & Open minded & $\begin{array}{l}\text { Explanation }(\mathrm{CE}= \\
\text { Madrid, } 80 €)\end{array}$ & $\begin{array}{c}\text { (Paris, } \\
100 €)\end{array}$ & Reject & Beginning & $70 \%$ \\
\hline$A C B_{i}$ & $\mathrm{AC2}$ & Open minded & $\begin{array}{c}\text { Threat }(\mathrm{Ac}=\text { see other } \\
\text { travel agency) }\end{array}$ & $\begin{array}{c}\text { (Paris, } \\
100 €)\end{array}$ & Reject & End & $90 \%$ \\
\hline
\end{tabular}


Message 4 contains a Support argument in which the support set has the features of the offer. $A_{i}$ computes her utility from this offer, and since she is not satisfied yet, she attacks it by sending a rebut argument (CE) in message 5 generated using her ACB (i.e., case $A C 1$ ). In message 6 the travel agency sends an Explanation argument with an AS, more precisely, an APO saying most of customers accept this offer. Message 8 and 9 present a Reward and a Threat respectively, where $A_{0}$ explained the missing feature (museums) and offered a reward. As for $A_{i}$ she threatened $A_{0}$ saying that she also wants a 4 stars hotel, otherwise, she will check another agency.

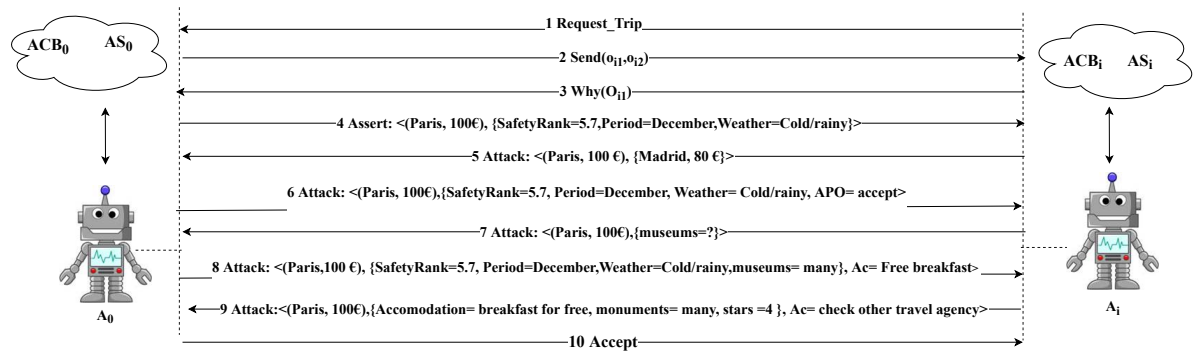

Fig. 2: An example of an ABN dialogue

\section{Conclusion}

This paper presents an Argumentation-Based Negotiation framework to aid the travel agency and its customers in their trip negotiations. In general, negotiations between a buyer and a seller are very strategic. The domain of tourism is not an exception, the travel agency seeks to maximize its profits and customers try to minimize their costs and maximize their satisfaction.

From this perspective, we presented the framework's architecture and the reasoning process that help agents to select the best argument to send at each part of the dialogue. In fact, a strategy is defined based on agents' profiles and their Arguments Case-Base. The travel agency and its customers are characterized by an Argumentative profile that presents their ways of reasoning. Customers are also characterized by a Preference profile that embodies their preferences regarding a trip. Both negotiation parties have their own Arguments Case-Base that they use to select the appropriate argument in a given situation.

Future work will focus on an experimental study in which more refined strategies will be proposed and tested for their impact on the negotiation process.

\section{References}

1. Aamodt, A., Plaza, E.: Case-based reasoning: Foundational issues, methodological variations, and system approaches. AI communications 7(1), 39-59 (1994) 
2. Adnan, M.H.M., Hassan, M.F., Aziz, I., Paputungan, I.V.: Protocols for agentbased autonomous negotiations: a review. In: ICCOINS. pp. 622-626. IEEE (2016)

3. Amgoud, L., Parsons, S.: Agent dialogues with conflicting preferences. In: ATAL. pp. 190-205. Springer (2001)

4. Amgoud, L., Prade, H.: Generation and evaluation of different types of arguments in negotiation. In: NMR. pp. 10-15 (2004)

5. Bouslama, R., Ayachi, R., Ben Amor, N.: A new generic framework for argumentation-based negotiation using case-based reasoning. In: IPMU. pp. 633644. Springer (2018)

6. Bouslama, R., Ayachi, R., Ben Amor, N.: A new generic framework for mediated multilateral argumentation-based negotiation using case-based reasoning. In: ECSQARU. pp. 14-26. Springer (2019)

7. Dimopoulos, Y., Moraitis, P.: Advances in argumentation based negotiation. Negotiation and argumentation in multi-agent systems: fundamentals, theories, systems and applications pp. 82-125 (2014)

8. Hadidi, N., Dimopoulos, Y., Moraitis, P.: Tactics and concessions for argumentation-based negotiation. Computational Models of Argument: Proceedings of COMMA 2012 245, 285-296 (2012)

9. Hadoux, E., Hunter, A.: Strategic sequences of arguments for persuasion using decision trees. In: AAAI (2017)

10. Heras, S., Jordán, J., Botti, V., Julián, V.: Argue to agree: a case-based argumentation approach. IJAR 54(1), 82-108 (2013)

11. Heras, S., Jordán, J., Botti, V., Julián, V.: Case-based strategies for argumentation dialogues in agent societies. Information Sciences 223, 1-30 (2013)

12. Jennings, N.R., Faratin, P., Lomuscio, A.R., Parsons, S., Sierra, C., Wooldridge, M.: Automated negotiation: prospects, methods and challenges. International Journal of Group Decision and Negotiation 10(2), 199-215 (2001)

13. Lazar, C.M.: Internet-an aid for e-tourism. Ecoforum Journal 8(1) (2019)

14. Lopes, F., Novais, A.Q., Coelho, H.: Bilateral negotiation in a multi-agent energy market. In: ICICA. pp. 655-664. Springer (2009)

15. Park, S., Tussyadiah, I., Mazanec, J., Fesenmaier, D.: Travel personae of american pleasure travelers: A network analysis. Journal of Travel \& Tourism Marketing 27, 797-811 (2010)

16. Rahwan, I., Ramchurn, S.D., Jennings, N.R., Mcburney, P., Parsons, S., Sonenberg, L.: Argumentation-based negotiation. KER 18(4), 343-375 (2003)

17. Rahwan, I., Sonenberg, L., McBurney, P.: Bargaining and argument-based negotiation: Some preliminary comparisons. In: ArgMAS. pp. 176-191. Springer (2004)

18. Sierra, C., Jennings, N.R., Noriega, P., Parsons, S.: A framework for argumentation-based negotiation. In: ATAL. pp. 177-192. Springer (1997)

19. Soh, L.K., Tsatsoulis, C.: Agent-based argumentative negotiations with case-based reasoning. In: AAAI Fall Symposium Series on Negotiation Methods for Autonomous Cooperative Systems. pp. 16-25 (2001)

20. Sycara, K.P.: Persuasive argumentation in negotiation. Theory and decision 28(3), 203-242 (1990)

21. Walton, D.: Argumentation schemes for presumptive reasoning. Routledge (2013) 